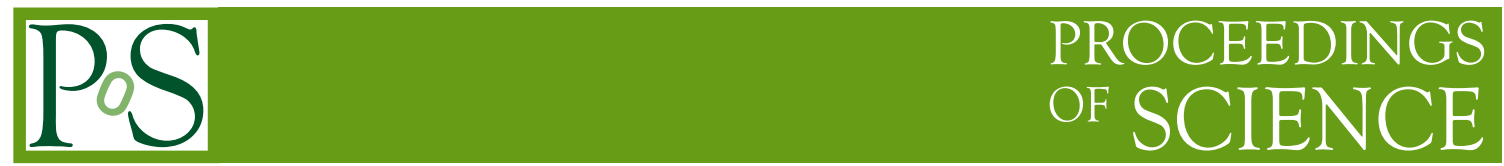

\title{
Neutrino Cosmology: Current Results
}

\author{
Massimiliano Lattanzi* \\ Istituto Nazionale di Fisica Nucleare, Sezione di Ferrara and Dipartimento di Fisica e Scienze \\ della Terra, Via Giuseppe Saragat 1, I-44122 Ferrara, Italy \\ E-mail: lattanzi@fe.infn.it
}

I review the current constraints on neutrino properties from available cosmological data, in particular from Planck observations of the cosmic microwave background anisotropies, and on BOSS measurements of galaxy clustering. I focus on constraints on the sum of neutrino masses, on the effective number of degrees of freedom (including light sterile neutrinos) and on non-standard neutrino interactions.

Neutrino Oscillation Workshop

4 - 11 September, 2016

Otranto (Lecce, Italy)

* Speaker. 


\section{Introduction}

The presence of a sea of relic neutrinos, i.e., of a cosmic neutrino background $(\mathrm{Cv} \mathrm{B})$, is a basic prediction of the standard $\Lambda \mathrm{CDM}$ model. In the early Universe, at temperatures $T>1 \mathrm{MeV}$, neutrinos were kept in equilibrium with the other species in the cosmological fluid by weak interactions. These cease to be effective at $T \sim 1 \mathrm{MeV}$; at this point neutrinos decouple from the rest of the fluid and start to propagate freely across the Universe, a regime known as "free streaming". At the present time, the $\mathrm{C} v \mathrm{~B}$ should have a thermal spectrum, with temperature $T_{v}=(4 / 11)^{1 / 3} T_{\mathrm{CMB}} \simeq 1.9 \mathrm{~K}$, corresponding to a number density of $\sim 113$ particles $/ \mathrm{cm}^{3}$. This picture is based on general relativity (applied to a spatially homogeneous and isotropic system) and on the standard model of particle physics (SM), with the additional assumption that the Universe at $T \sim$ few $\mathrm{MeV}$ was already in thermal equilibrium. A direct detection of the $\mathrm{C} v \mathrm{~B}$ is a challenging task, that probably will not be achieved in the near future; however, the picture just described is strongly supported by observations, as we shall see in more detail in the following. In particular, the cosmological energy density of relativistic particles other than photons, as inferred from observations of the cosmic microwave background (CMB) radiation, is in agreement (within an uncertainty of $\sim 10 \%$ ) with the value expected for three neutrino species with $T_{V}=1.9 \mathrm{~K}$ [1]. Moreover, observations do not suggest any departure from the free streaming regime, or from a thermal spectrum.

Neutrinos affect the cosmological evolution at both the background (i.e., the expansion history) and perturbation level (see [2] for a detailed review). Background effects include changes in the time of matter-radiation equality or in the distance to the last scattering surface; these are usually "large" effects (when compared with current instrumental sensitivities), but can be compensated by acting on other parameters of the $\Lambda \mathrm{CDM}$ model. Thus their constraining power is limited by parameter degeneracies. On the other hand, the effect of neutrinos on perturbation evolution is quite peculiar, in that free-streaming leads to a suppression of perturbations below a critical scale, corresponding to the Hubble radius at the time when neutrinos become non-relativistic. This is due to the fact that, given their large velocity dispersion $(\sim c / \sqrt{3}$ in the ultrarelativistic regime), they can escape potential wells even at the scale of the horizon. Well below the free-streaming scale, the power suppression is proportional to the fraction of matter density provided by neutrinos. Moreover, the growth of matter perturbations is also slower in presence of free-streaming neutrinos.

Given their effect on the cosmological evolution, neutrino properties (like their mass) can be constrained by cosmological observations, and in my talk at NOW 2016 I have reviewed the current constraints on neutrino properties coming from the available cosmological data. The observed pattern of CMB anisotropies allows to measure precisely the parameters of the $\Lambda \mathrm{CDM}$ model, while tightly constraining possible deviations from the model itself, and represent a powerful probe of neutrino physics. To date, the most precise measurements of CMB anisotropies in both temperature and polarization, on a wide range of scales, are those provided by the Planck satellite [3], that imaged the whole microwave sky with an unprecedented combination of sensitivity, angular resolution and frequency coverage. The second release of cosmological data from Planck has taken place in early 2015 (see Ref. [3] for an overview of the scientific products); the final, legacy release is expected in the near future. The Planck data from the 2015 public release represent the baseline dataset used to derive the constraints reported here and are described in detail in Refs. [4]. The 
baseline combination, denoted "Planck $T T+$ lowP", considers the Planck temperature power spectrum in the whole multipole range $2 \leq \ell \leq 2500$, and the "low- $\ell$ " (i.e., $\ell<30$ ) polarization. The inclusion of $T E$ and $E E$ polarization information at $\ell \geq 30$, resulting in the dataset dubbed "Planck $T T, T E, E E$ + lowP", further increases the constraining power; however, since small-scale polarization could be affected by low-level residual systematics, the results obtained using these data should be regarded as non-conservative. Finally, the measurement of the lensing potential as reconstructed from the Planck maps of temperature and polarization anisotropies is denoted simply as "lensing". The analysis made by the Planck collaboration also considers auxiliary, external datasets, in particular a compilation of baryon acoustic oscillations (BAO) measurements [5] (simply denoted "BAO"), observations of Type IA supernovae [6], and astrophysical estimates of the Hubble constant [7]; these are collectively denoted as "external". The cosmological implications of the 2015 Planck data release are reported in Ref. [1].

The constraining power of CMB observations is significantly enhanced when combined with geometrical information, like that coming from BAO, or direct measurements of the Hubble constant, since it allows to break parameter degeneracies. Clustering information, like that coming from direct observations of the distribution of galaxies, or from weak lensing effects (like e.g. galaxy or CMB weak lensing), that measure the gravitational potential integrated along the line of sight, is also very useful in the context of neutrino cosmology, given the effect of neutrinos on the clustering of matter described above. Recently, the final galaxy clustering dataset from the Baryon Oscillation Spectroscopic Survey (BOSS) [8] was released, based on the Data Release 12 (DR12) of the Sloan Digital Sky Survey III [9]. The cosmological analysis of the DR12 galaxy sample is presented in Ref. [10], and uses, in combination with Planck 2015 data, BOSS's own information on the BAO feature and on the full shape of the galaxy power spectrum (here dubbed "BAO-12" and "FS-12", respectively), and possibly external information based on direct measurements of the Hubble constant from Ref. [11].

The paper is organized as follows: in Sec. 2 I report current constraints on neutrino masses, and in Sec. 3 those on the effective number of degrees of freedom (including constraints on light sterile neutrinos). In Sec. 4 I briefly discuss the agreement of the behaviour of neutrino perturbations with the standard expectations. In the following, unless otherwise specified, I report $68 \% \mathrm{CL}$ uncertainties. Results reported as obtained by the "Planck collaboration" and the "BOSS collaboration" are taken from Refs. [1] and [10], respectively.

\section{Constraints on the sum of neutrino masses}

By themselves, CMB primary anisotropies (those generated at the time of decoupling) are directly sensitive only to individual neutrino masses larger than roughly $0.6 \mathrm{eV}$, since for smaller masses the transition to the non-relativistic regime takes place after CMB decoupling. However, this limitation can be overcome by precise measurements of small-scale anisotropies, that are affected by the lensing due to the intervening matter distribution between us and the last scattering surface. Lensing leaves an imprint in the temperature and polarization power spectra; in addition, the spectrum of the lensing potential itself can be reconstructed from the higher-order moments of the anisotropy pattern. In fact, Planck sensitivity to the weak lensing of CMB photons allows to get upper bounds on the sum on neutrino masses $M_{v}$ in the sub-eV range, as it can be seen in Tab. 
1, where we report the limits obtained by the Planck collaboration. The baseline Planck $T T+$ lowP dataset gives $M_{v}<0.72 \mathrm{eV}$ (unless otherwise stated, upper limits are at 95\% C.L.), while inclusion of small-scale polarization information improves the constraint to $M_{v}<0.49 \mathrm{eV}^{1}$. Including $\mathrm{BAO}$ or other external data greatly adds to the constraining power, bringing the limits down in the $0.2 \mathrm{eV}$ ballpark, by breaking geometrical degeneracies; in particular, the PlanckTT+lowP+BAO dataset combination yields $M_{v}<0.21 \mathrm{eV}$. Even tighter constraints can be obtained by adding information on the matter distribution. The BOSS collaboration, using their own measurements of BAO and of the full shape of the matter power spectrum from the DR12 data release, in combination with Planck 2015 data, obtains $M_{v}<0.16 \mathrm{eV}$ (Planck + BAO-12 + FS-12). In Ref. [13] , the BOSS Lyman- $\alpha$ forest measurements are used, again together with Planck 2015 data, to get $M_{V}<0.12 \mathrm{eV}$. It should be noted however that measurements of the shape of the matter power spectrum could be affected by systematics (like e.g. those related to the nonlinear evolution of perturbations), so that these results should be regarded as less conservative. Even more care should be taken in interpreting the Ly- $\alpha$ forest measurements. Very tight $(\sim 0.12 \mathrm{eV})$ bounds can also be obtained by combining CMB and galaxy power spectrum data with direct $H_{0}$ measurements; however these results are at least partly driven by tensions between datasets (see also discussion in the next session).

Neutrino masses can also be constrained by laboratory experiments. Oscillation experiments only measure the mass differences, but not the absolute mass scale; however, they allow to put lower limits on the sum of neutrino masses. In particular, $M_{v} \geq 0.06 \mathrm{eV}$ for normal hierarchy $(\mathrm{NH})$, and $M_{v} \geq 0.1 \mathrm{eV}$ for inverted hierarchy (IH). Given these values, it is clear how the combination of present $\mathrm{CMB}$ and BAO measurements from Planck and BOSS is starting to probe the region in which the two hierarchies could in principle be distinguished (see e.g. Ref. [14] for a statistically robust assessment of the sensitivity of cosmological data to the mass hierarchy).

Table 1: Planck constraints on the total neutrino mass $M_{v}$, for different datasets combination (limits are at 95\% CL).

\begin{tabular}{|c|c|c|c|c|c|}
\hline Dataset & & + lensing & $+\mathrm{BAO}$ & + ext & + lensing + ext \\
\hline Planck TT + lowP & $<0.72 \mathrm{eV}$ & $<0.68 \mathrm{eV}$ & $<0.21 \mathrm{eV}$ & $<0.20 \mathrm{eV}$ & $<0.23 \mathrm{eV}$ \\
\hline Planck TT, TE, EE + lowP & $<0.49 \mathrm{eV}$ & $<0.59 \mathrm{eV}$ & $<0.17 \mathrm{eV}$ & $<0.15 \mathrm{eV}$ & $<0.19 \mathrm{eV}$ \\
\hline
\end{tabular}

\section{Effective numbers of degrees of freedom}

Given the thermal history described in the introduction, the contribution of neutrinos to the energy density in relativistic species in the $\Lambda \mathrm{CDM}$ model is fixed: in the limit of instantaneous decoupling, one expects an energy density $\rho_{v}^{\text {std }}=\left(7 \pi^{2} / 120\right)(4 / 11)^{4 / 3} T_{\mathrm{CMB}}^{4}$ per each neutrino family.

\footnotetext{
${ }^{1}$ In 2016, the Planck collaboration has reported a new estimate of the optical depth to reionization $\tau$, base on new, not yet public, low- $\ell$ polarization spectra [12]. Due to parameter degeneracies, the CMB constraints from PlanckTT and the 2016 low- $\ell$ polarization tighten to $M_{V}<0.59 \mathrm{eV}$.
} 
The effective number of neutrino families $N_{\text {eff }}$ is defined as the ratio between the total density of relativistic species (excluding photons), and $\rho_{v}^{\text {std }}$. The standard expectation, corresponding to the contribution of the three standard model neutrinos, is $N_{\text {eff }}=3.046$ (this value has been recently updated to $N_{\text {eff }}=3.045$ in Ref. [15]); the value is not exactly equal to 3 due non-instantaneous decoupling [16]. A deviation from $N_{\text {eff }}=3.046$ (usually parameterized in terms of $\Delta N_{\text {eff }} \equiv N_{\text {eff }}-3.046$ ) could be due to the presence of some extra relativistic degree of freedom in the early Universe, like a fourth light neutrino eigenstate, or some other light particle species not necessarily related to neutrinos (like e.g., a thermal axion). A non-thermal spectrum for neutrinos (like in low-reheating scenarios, in which $\Delta N_{\text {eff }}<0$ ), as well a non-zero chemical potential, would also result in a nonstandard value for $N_{\text {eff }}$, other than having more subtle effects related to the different velocity dispersion (and in general to the different shape of the distribution function).

Increasing $N_{\text {eff }}$, while keeping fixed both the time of matter-radiation equality and the distance to the last scattering surface, has the effect of suppressing the high- $\ell$ tail of the CMB (and viceversa), due to the increased photon damping. The value of $N_{\text {eff }}$ currently inferred from CMB observations is in agreement with $N_{\text {eff }}=3.046$, within the experimental uncertainty. Results obtained by the Planck collaboration, using Planck data also in combination with BAO, are shown in Tab. 2 (adding lensing has little effect on the constraints). Even in the most conservative case (PlanckTT+lowP: $N_{\text {eff }}=3.13 \pm 0.32$ ), $\Delta N_{\text {eff }}=1$ is excluded at the $3 \sigma$ level; adding BAO information reduces the uncertainty by roughly $30 \%$, making the exclusion even more significant. The BOSS collaboration, using Planck + BAO- $12+$ FS-12 data, finds $N_{\text {eff }}=3.03 \pm 0.18$. It should be noted that using astrophysical measurements of the Hubble constant $H_{0}$ as a geometrical probe, in combination with Planck CMB data, results in higher values of $N_{\text {eff }}$ (for example, adding to the combined Planck and BOSS dataset a prior $H_{0}=73.0 \pm 1.8 \mathrm{~km} \mathrm{~s} \mathrm{Mpc}^{-1}$ based on the measurements reported in Ref. [11], yields $N_{\text {eff }}=3.18 \pm 0.16$ ). This is due to the tension between the astrophysical values of $H_{0}$ and the value inferred from CMB (and BAO) in the framework of $\Lambda \mathrm{CDM}$. Given the well-known degeneracy between $H_{0}$ and $N_{\text {eff }}$ (the angular size subtended by the sound horizon at recombination, accurately measured by Planck, can be kept constant by simultaneously increasing or decreasing both parameters), this tension is alleviated in models with $\Delta N_{\text {eff }}>0$. Unfortunately, increasing $N_{\text {eff }}$ exacerbates another tension, namely that between the values of the fluctuation amplitude inferred by Planck on one side and by large-scale structure data on the other.

The limits just reported have been obtained in a one-parameter extension of $\Lambda$ CDM in which $N_{\text {eff }}$ is treated as a free parameter. This can be seen as the limit in which the additional species contributing to $\Delta N_{\text {eff }}$, for example a sterile neutrino, is effectively massless. For massive species, the full form of the distribution function should be in principle be specified; however, an effective parameterization in terms of $\Delta N_{\text {eff }}$ and of an effective mass $m_{s}^{\text {eff }}$ allows to cover several physically interesting cases [17]. These include the case of a fourth massive neutrino eigenstate that either (i) has a thermal distribution with arbitrary temperature $T_{s}$, or (ii) is distributed proportionally to the active neutrinos, but with a suppression factor $\chi_{s}$ (this corresponds to the Dodelson-Widrow (DW) prediction for the non-resonant production scenario [18]; see also Ref. [19]). The actual mass $m_{s}$ of the sterile can be recovered from the parameters of the "effective model" by means of $m_{s}=\left(T_{s} / T_{v}\right)^{-3} m_{s}^{\text {eff }}=\Delta N_{\mathrm{eff}}^{-3 / 4} m_{s}^{\text {eff }}$ for the thermal case, and $m_{s}=\chi_{s}^{-1} m_{s}^{\text {eff }}=\Delta N_{\mathrm{eff}}^{-1} m_{s}^{\text {eff }}$ for the DW case. Planck is consistent with no sterile neutrinos: the $95 \%$ allowed region in parameter space is 
$N_{\text {eff }}<3.7, m_{s}^{\text {eff }}<0.52 \mathrm{eV}$ from PlanckTT + lowP + lensing + BAO.

A fourth light neutrino eigenstate has been advocated as a possible explanation of the anomalies observed in short-baseline (SBL) experiments (see e.g. Ref. [20] and references therein). However, it has been shown that a sterile neutrino with the mass $\left(m_{s} \simeq 1 \mathrm{eV}\right)$ and coupling required to explain reactor anomalies would rapidly thermalize in the early Universe (see e.g. Refs. [21]) and lead to $\Delta N_{\text {eff }}=1$, strongly at variance with cosmological constraints (excluded at more than $99 \%$ confidence considering the above combination of Planck and BAO data). Possible mechanisms to avoid full thermalization, and thus reconcile the sterile neutrino solution to SBL anomalies with cosmological observations include large lepton asymmetries, new interactions, or particle decays (see e.g. [22], as well as references in [20]).

Table 2: Planck constraints on the effective number of neutrino families $N_{\text {eff }}$, for different datasets combination (68\% CL uncertainties).

\begin{tabular}{lll} 
Dataset & & $+\mathrm{BAO}$ \\
\hline Planck TT + lowP & $3.13 \pm 0.32$ & $3.15 \pm 0.23$ \\
Planck TT, TE, EE + lowP & $2.99 \pm 0.20$ & $3.04 \pm 0.18$ \\
\hline
\end{tabular}

\section{Non-standard neutrino interactions}

The $\Lambda \mathrm{CDM}$ prediction of free-streaming neutrinos can also be tested by cosmological observations. This can be done in different ways: the approach followed by the Planck collaboration is to consider the effective neutrino sound speed $c_{\text {eff }}$ and viscosity $c_{\text {vis }}$ [23] and look for deviations from the standard values $c_{\mathrm{eff}}^{2}=c_{\mathrm{vis}}^{2}=1 / 3$ expected for free-streaming particles. In this approach the parameters are constant in time. Alternatively, one can model the transition from a non-collisional to a collisional regime (or viceversa) either by abruptly switching between the two regimes at some critical redshit [24], or by directly taking into account collisional processes in the Boltzmann evolution for neutrinos [25, 26], consistently with the underlying particle physics model. The latter two approaches allow for example to differentiate the cases of neutrino interactions mediated by a scalar or vector particle.

This is an interesting test as it allows to test the robustness of the base $\Lambda \mathrm{CDM}$ model and at the same time to probe for non-standard physics in the neutrino sector, like neutrino interactions beyond the standard model of particle physics (for example, those mediated by majorons). Planck data are fairly consistent with free-streaming neutrinos: using the $\left(c_{\text {eff }}, c_{\text {vis }}\right)$ approach, the Planck collaboration finds $c_{\text {eff }}^{2}=0.312 \pm 0.011$ and $c_{\text {vis }}^{2}=0.47_{-0.12}^{+0.26}$ from PlanckTT+lowP; inclusion of the small-scale Planck polarization data strengthens the agreement. Introducing instead neutrino scattering by means of an effective scalar neutrino coupling $g_{\text {eff }}$, it is found that the Planck data are consistent with $g_{\text {eff }}=0$ [26]; moreover, upper limits on $g_{\text {eff }}$ are competitive with those that can be obtained through other cosmological and astrophysical probes, like e.g. supernovae. 


\section{Summary}

I have shown how cosmological data, in particular CMB observations from Planck and galaxy clustering observations from BOSS, can be used to constrain neutrino properties. Present data provide tight bounds on the sum of neutrino masses, while at the same time they do not show evidence for new physics beyond the standard model of particles. The presence of an additional massless, fully thermalized species is excluded, thus ruling out with high confidence the preferred sterile neutrino solution to the SBL anomalies, at least in its simplest implementation.

\section{References}

[1] P. A. R. Ade et al. [Planck Collaboration], Astron. Astrophys. 594, A13 (2016).

[2] J. Lesgourgues and S. Pastor, Phys. Rept. 429, 307 (2006); J. Lesgourgues and S. Pastor, New J. Phys. 16, 065002 (2014);

[3] R. Adam et al. [Planck Collaboration], Astron. Astrophys. 594, A1 (2016).

[4] N. Aghanim et al. [Planck Collaboration], Astron. Astrophys. 594, A11 (2016); P. A. R. Ade et al. [Planck Collaboration], Astron. Astrophys. 594, A15 (2016).

[5] F. Beutler et al., Mon. Not. Roy. Astron. Soc. 416, 3017 (2011); A. J. Ross, L. Samushia, C. Howlett, W. J. Percival, A. Burden and M. Manera, Mon. Not. Roy. Astron. Soc. 449, no. 1, 835 (2015);

L. Anderson et al. [BOSS Collaboration], Mon. Not. Roy. Astron. Soc. 441, no. 1, 24 (2014).

[6] M. Betoule et al. [SDSS Collaboration], Astron. Astrophys. 568, A22 (2014).

[7] G. Efstathiou, Mon. Not. Roy. Astron. Soc. 440, no. 2, 1138 (2014); E. M. L. Humphreys, M. J. Reid, J. M. Moran, L. J. Greenhill and A. L. Argon, Astrophys. J. 775, 13 (2013).

[8] K. S. Dawson et al. [BOSS Collaboration], Astron. J. 145, 10 (2013).

[9] S. Alam et al. [SDSS-III Collaboration], Astrophys. J. Suppl. 219, 12 (2015); B. Reid et al., Mon. Not. Roy. Astron. Soc. 455, 1553 (2016)

[10] S. Alam et al. [BOSS Collaboration], [arXiv:1607.03155 [astro-ph.CO]].

[11] A. G. Riess et al., Astrophys. J. 826, 56 (2016).

[12] N. Aghanim et al. [Planck Collaboration], Astron. Astrophys. 596, A107 (2016).

[13] N. Palanque-Delabrouille et al., JCAP 1511, 011 (2015).

[14] M. Gerbino, M. Lattanzi, O. Mena and K. Freese, arXiv:1611.07847 [astro-ph.CO].

[15] P. F. de Salas and S. Pastor, JCAP 1607, 051 (2016).

[16] A. D. Dolgov, S. H. Hansen and D. V. Semikoz, Nucl. Phys. B 543, 269 (1999); G. Mangano, G. Miele, S. Pastor and M. Peloso, Phys. Lett. B 534, 8 (2002).

[17] S. Colombi, S. Dodelson and L. M. Widrow, Astrophys. J. 458, 1 (1996).

[18] S. Dodelson and L. M. Widrow, Phys. Rev. Lett. 72, 17 (1994).

[19] A. Merle, A. Schneider and M. Totzauer, JCAP 1604, no. 04, 003 (2016).

[20] S. Gariazzo, C. Giunti, M. Laveder, Y. F. Li and E. M. Zavanin, J. Phys. G 43, 033001 (2016). 
[21] A. Mirizzi, G. Mangano, N. Saviano, E. Borriello, C. Giunti, G. Miele and O. Pisanti, Phys. Lett. B 726, 8 (2013); S. Hannestad, R. S. Hansen, T. Tram and Y. Y. Y. Wong, JCAP 1508, 019 (2015).

[22] N. Saviano, A. Mirizzi, O. Pisanti, P. D. Serpico, G. Mangano and G. Miele, Phys. Rev. D 87, 073006 (2013); N. Saviano, O. Pisanti, G. Mangano and A. Mirizzi, Phys. Rev. D 90, 113009 (2014); M. Archidiacono, S. Gariazzo, C. Giunti, S. Hannestad, R. Hansen, M. Laveder and T. Tram, JCAP 1608, 067 (2016).

[23] W. Hu, Astrophys. J. 506, 485 (1998).

[24] M. Archidiacono and S. Hannestad, JCAP 1407, 046 (2014).

[25] F. Y. Cyr-Racine and K. Sigurdson, Phys. Rev. D 90,123533 (2014).

[26] F. Forastieri, M. Lattanzi and P. Natoli, JCAP 1507, 014 (2015). 